\title{
The Triage Effectiveness of an Extended High-Risk Human Papillomavirus Genotyping Assay for Women with Cytology Showing Atypical Squamous Cells of Undetermined Significance in China
}

This article was published in the following Dove Press journal:

Risk Management and Healthcare Policy

\author{
Diling Pan $\mathbb{D}^{1, *}$ \\ Binhua Dong $\mathbb{D}^{2,3, *}$ \\ Hangjing Gao iD ${ }^{2}$ \\ Xiaodan Mao iD ${ }^{2,3}$ \\ Huifeng Xue ${ }^{4}$ \\ Pengming Sun (D) 2,3 \\ 'Department of Pathology, Fujian \\ Maternity and Child Health Hospital, \\ Affiliated Hospital of Fujian Medical \\ University, Fuzhou 35000I, Fujian, \\ People's Republic of China; ${ }^{2}$ Department \\ of Gynecology, Laboratory of \\ Gynecologic Oncology, Fujian Maternity \\ and Child Health Hospital, Affiliated \\ Hospital of Fujian Medical University, \\ Fuzhou, Fujian 35000I, People's Republic \\ of China; ${ }^{3}$ Fujian Key Laboratory of \\ Women and Children's Critical Diseases \\ Research, Fujian Maternity and Child \\ Health Hospital, Affiliated Hospital of \\ Fujian Medical University, Fuzhou, Fujian \\ 35000 I, People's Republic of China; \\ ${ }^{4}$ Fujian Provincial Cervical Disease \\ Diagnosis and Treatment Health Center, \\ Fujian Maternity and Child Health \\ Hospital, Affiliated Hospital of Fujian \\ Medical University, Fuzhou 35000I, \\ Fujian, People's Republic of China \\ *These authors contributed equally to \\ this work
}

Correspondence: Pengming Sun Fujian Maternity and Child Health Hospital, Affiliated Hospital of Fujian Medical University, 18 Daoshan Road, Fuzhou 35000 I, Fujian, People's Republic of China Tel +86-59l-87558732

Fax +86-59I-8755I247

Email sunfemy@hotmail.com
Purpose: Little is known about the performance of extended high-risk human papillomavirus (HR-HPV) genotyping triage of cytology showing atypical squamous cells of undetermined significance (ASC-US). This study aims to evaluate the effectiveness of triage with different HR-HPV genotype models among women with ASC-US.

Materials and Methods: In this study, all women who underwent cervical cytology and HR-HPV genotyping were enrolled from 2014 to 2017 in China, and those with cytology showing ASC-US were referred for colposcopy and/or biopsy. The endpoint was histological detection of cervical intraepithelial neoplasia grade 2 or worse (CIN2+). The outcome indicators were the sensitivity, specificity, positive predictive values (PPVs), negative predictive values (NPVs) and colposcopy referral rates.

Results: In all, 56,788 women were enrolled in this study, and 2658 (4.97\%) women were reported to have ASC-US; 10.1\% (242/2393) of women with ASC-US were identified as having CIN2+. The HR-HPV infection rate was $95.0 \%$ among all women with ASC-US who were identified as $\mathrm{CIN} 2+$, and the top five genotypes with prevalence and risk of CIN2+ were HPV16 (OR=26.38), HPV58 (OR=7.04), HPV18 (OR=4.44), HPV33 (OR=3.38), HPV31 $(\mathrm{OR}=2.97)$ and HPV52 (OR=2.96). The HPV16/18/31/33/52/58 model achieved higher sensitivity [91.3 (87.8-94.9)], specificity [70.0 (68.1-72.0)], PPV [25.5 (22.4-28.2)] and NPV [98.6 (97.3-98.7)] for the triage of ASC-US patients than the other HR-HPV-type combination models, but the colposcopy referral rate $(36.2 \%)$ was significantly lower than that of the recommended HR-HPV nongenotyping model (47.6\%).

Conclusion: This study confirms that the specific HR-HPV genotype HPV16/18/31/33/52/ 58 is an alternative strategy for ASC-US triage and can effectively reduce the high burden of colposcopy referrals in China.

Keywords: human papillomavirus, genotyping, atypical squamous cells of undetermined significance, cervical intraepithelial neoplasia

\section{Introduction}

Cervical cancer remains a high burden on global public health, with 530,000 new cases of cervical cancer diagnosed worldwide each year, ${ }^{1}$ however, cervical cancer is relatively easy to prevent by screening. ${ }^{2}$ Thus, it is particularly important to optimize screening programs to reduce the burden of cervical cancer. When atypical transformation is detected by cytology, further treatment and/or follow-up may be 
needed based on the severity of the lesion. ${ }^{3,4}$ However, it remains a pending issue for the management strategy of women with atypical squamous cells of undetermined significance (ASC-US). ${ }^{5}$ ASC-US is not a specific diagnosis of progression or disease; rather, this indistinct diagnosis encompasses normal, low-grade squamous intraepithelial lesions (LSILs), high-squamous intraepithelial lesions (HSILs) and invasive cancer. ${ }^{6}$ In 2020, the American Society for Colposcopy and Cervical Pathology recommended immediate HPV testing, but 12-month repeat cytology was considered acceptable. ${ }^{7}$ In recent years, in many countries including the United States, immediate HPV testing is recommended as the preferred strategy after cytology showing ASC-US. ${ }^{8}$ Although most cases of ASC-US resolve with no apparent clinical disease, $15-20 \%$ are confirmed by histology to be cervical epithelial neoplasia (CIN) during follow-up. ${ }^{9}$ Previous research has indicated that the risk of CIN3 or worse among women with ASC-US is significantly higher than that among women without ASC-US. ${ }^{10}$ Therefore, an accurate category for these patients is needed to determine who needs subsequent management.

Research has confirmed that persistent infection with HRHPV is a major factor in the development of cervical cancer. ${ }^{11,12}$ In recent years, HR-HPV testing has been recommended for the management of women with ASC-US, and colposcopy is recommended for all HR-HPV-positive cases. ${ }^{13}$ However, this management strategy may be overtreatment; ${ }^{13}$ because of the different carcinogenicities of the different HPV genotypes, the role of HPV in the development of CIN and cervical cancer varies with HPV genotype. The types of HPV infection related to cervical cancer are HPV16, 18, 31, 33, 35, 39, 45, 51, 52, 56, 58, 59, 66 and $68 .{ }^{14}$ Infection with HPV16 and HPV18 was found in $71 \%$ of women with invasive cervical cancer. ${ }^{15} \mathrm{~A}$ previous study ${ }^{16}$ indicated that the incidence of CIN2 or worse (CIN2+) at a follow-up of 13.4 years among HPV-infected women with initially normal cytology was as follows: $28.5 \%$ for those with HPV16 alone, $15.4 \%$ for those with HPV 18 alone, $19.1 \%$ for those with HPV $33,18.2 \%$ for those with HPV35, 16.7\% for those with HPV58, 15.7\% for those with HPV $31,8.6 \%$ for those with HPV $51,8.5 \%$ for those with HPV45, 4.7\% for those with HPV52, 3.6\% for those with HPV39, and $2.8 \%$ for those with HPV56. These data provide a reference for triaging patients with ASC-US to select appropriate HPV genotypes.

The ideal triage strategy for patients with ASC-US is to maximize the sensitivity and specificity, thereby reducing the rate of misdiagnosis and unnecessary colposcopy.
Herein, our research was conducted to examine the performance of different HPV genotype combined models for women with ASC-US prior to the detection of CIN2+ and offers new parameters to design an optimal strategy for the triage of patients with ASC-US.

\section{Materials and Methods \\ Study Subjects}

A cervical cancer screening group of 56,788 women was set up from January 2014 to December 2017 in Fujian Province. All participants underwent cervical cytology and HR-HPV genotyping assessments. Women who met the following criteria were enrolled in the research: patients aged 21 years or older; those with a history of sexual activity; those with cytology and HR-HPV genotyping for cervical cancer screening; and those who signed informed consent. Women who met the following criteria were excluded: those with a history of hysterectomy, cervical surgery, or cervical cancer treatment; those who are currently pregnant; those with severe immunodeficiency disease; those who had undergone cytology and HR-HPV testing within three years; and those who refused to sign the informed consent. Among them, women with ASC-US confirmed by cytology who underwent colposcopy and/or biopsy were finally eligible for our study. The Ethics Committees of the Fujian Maternity and Child Health Hospital granted approval for this study (2014-045). All participants in this study provided written informed consent.

\section{Screening Protocols}

Prior to registration, informed consent was obtained from each included woman. Then, a confidential questionnairebased interview was performed by an experienced doctor to evaluate each patient's medication and surgical history for cervical lesions; education level; history of smoking and drinking; and fertility history.

All qualified participants underwent gynecological examinations of the vulva, vagina and cervix. A doctor used a speculum to examine and collect cervical cells with plastic brushes. The cervical cells were collected in ThinPrep ${ }^{\circledR}$ Pap Test PreservCyt ${ }^{\circledR}$ Solution (Hologic Inc., Madison, WI, USA). A ThinPrep ${ }^{\circledR}$ Cytologic Test and HRHPV genotyping test were performed on ThinPrep cervical cells. If the liquid-based cytology test (TCT) results showed ASC-US, colposcopy and biopsy/ECC were performed within 10 weeks. A completely visible cervical 
transition area indicated satisfactory colposcopy; cervical biopsy was performed directly on visible lesions for women with abnormal colposcopy results; if colposcopy proved normal, a biopsy was not required. If colposcopy was unsatisfactory (the cervical transition area was not completely visible), endocervical curettage (ECC) was subsequently conducted. All enrolled participants were followed up for 12 months (Figure 1).

\section{HR-HPV Genotyping Assay}

The HR-HPV genotyping assay was performed using a PCR-reverse dot blot HPV genotyping kit (Yaneng Bioscience Co., Ltd., China) according to the instructions of manufacturer. The test can identify and distinguish 14 types of HR-HPV DNA in cervical exfoliated cells to detect each genotype based on nucleic acid amplification polymerase chain reaction (PCR) and point hybridization.

\section{Cytology and Histology}

The TCT and Bethesda System (TBS) were applied for cytology testing, and the CIN classification system was used for histology. For all histological results, the worst result was defined as the final result for each woman. If no biopsy was performed, or if the histological result was negative, the woman was regarded as CIN negative. Cell pathologists and histopathologists of Fujian Maternity and Child Health Hospital prepared and read the films. A session was convened to standardize the treatment protocols of colposcopy and histopathological diagnosis before the beginning of the study.

\section{Statistical Analysis}

Analyses were carried out with SPSS version 24.0 (IBM Corporation, New York, USA). The number of HR-HPVpositive patients with ASC-US divided by the total number of patients with ASC-US was regarded as the referral rate. The mean and standard deviation were calculated for the categorical variables. Values and percentages were also reported. We calculated the odds ratio (OR) of age and different HPV types to evaluate the risk of CIN2+ among women with ASC-US. We combined the HPV genotype in turn according to the OR to build the HR-HPV genotyping model. The sensitivity, specificity, positive predictive value (PPV), and negative predictive value (NPV) of the HR-HPV genotyping model for CIN2+ were evaluated and compared with the gold standard of pathological diagnosis. All of these results were used to assess the performance of
HR-HPV genotypes in the triage of women with ASC-US. The significance level was set at a P-value $<0.05$.

\section{Results \\ Characteristics for Women with Cytology Showing ASC-US}

Of the 56,788 women recruited, 53,464 were eligible and included in the study, $2658(5.0 \%)$ had confirmed ASC-US by cytology, $265(0.5 \%)$ refused colposcopy and/or biopsy and were excluded, and the remaining 2393 (4.5\%) who underwent colposcopy and/or biopsy were finally enrolled in this study. The characteristics of patients with ASC-US are shown in Table 1. The mean age of the women at enrollment was $38.4 \pm 10.3$ years (range, 21 to 73 years). Approximately $75.3 \%$ had completed middle school or higher education, $97.5 \%$ of those enrolled denied having a history of smoking, $68.4 \%$ denied having a history of drinking, $35.7 \%$ had more than 2 pregnancies, and $14.7 \%$ had more than 2 deliveries (Table 1).

\section{Prevalence Rates of the Different HPV Genotypes Among Women with ASC-US According to Cervical Pathologic Severity}

Table 2 contains data on the incident rates of the different HPV genotypes among women with ASC-US. The HR-HPV infection rate of ASC-US women aged 21-30 years was 51.2\%, and the HR-HPV infection rate gradually decreased with age, with the lowest rate of $40.7 \%$ among ASC-US women $>65$ years. The prevalence of HR-HPV among women with ASCUS was 47.6\% (1139/2393). HPV52 was the most prevalent, having been detected in 329 women $(13.7 \%)$, followed by HPV16 (226/2393, 9.4\%), HPV58 (201/2393, 8.4\%), HPV51 (103/2393, 4.3\%) and HPV18 (88/12393, 3.7\%).

The rate of HR-HPV infection increased with the severity of the pathological diagnosis. The prevalence rate of HRHPV was 32.6\% among women with normal pathology and $97.6 \%$ among women with CIN3 or cancer (Supplementary Table S1). Table 3 shows the distribution of different HRHPV genotype models according to the histologic diagnosis of CIN2+ in women with ASC-US. The rate of HR-HPV infection (95.0\%) in women with ASC-US with biopsyconfirmed CIN2+ was significantly higher than that in women with biopsy-confirmed negative for intraepithelial lesion or malignancy (NILM)/CIN1 (42.3\%). Among the women with ASC-US and biopsy-confirmed CIN2+, the prevalence rates of HR-HPV genotypes were as follows: HPV16, 47.9\%; HPV58, 21.1\%; HPV52, 18.6\%; HPV18, 


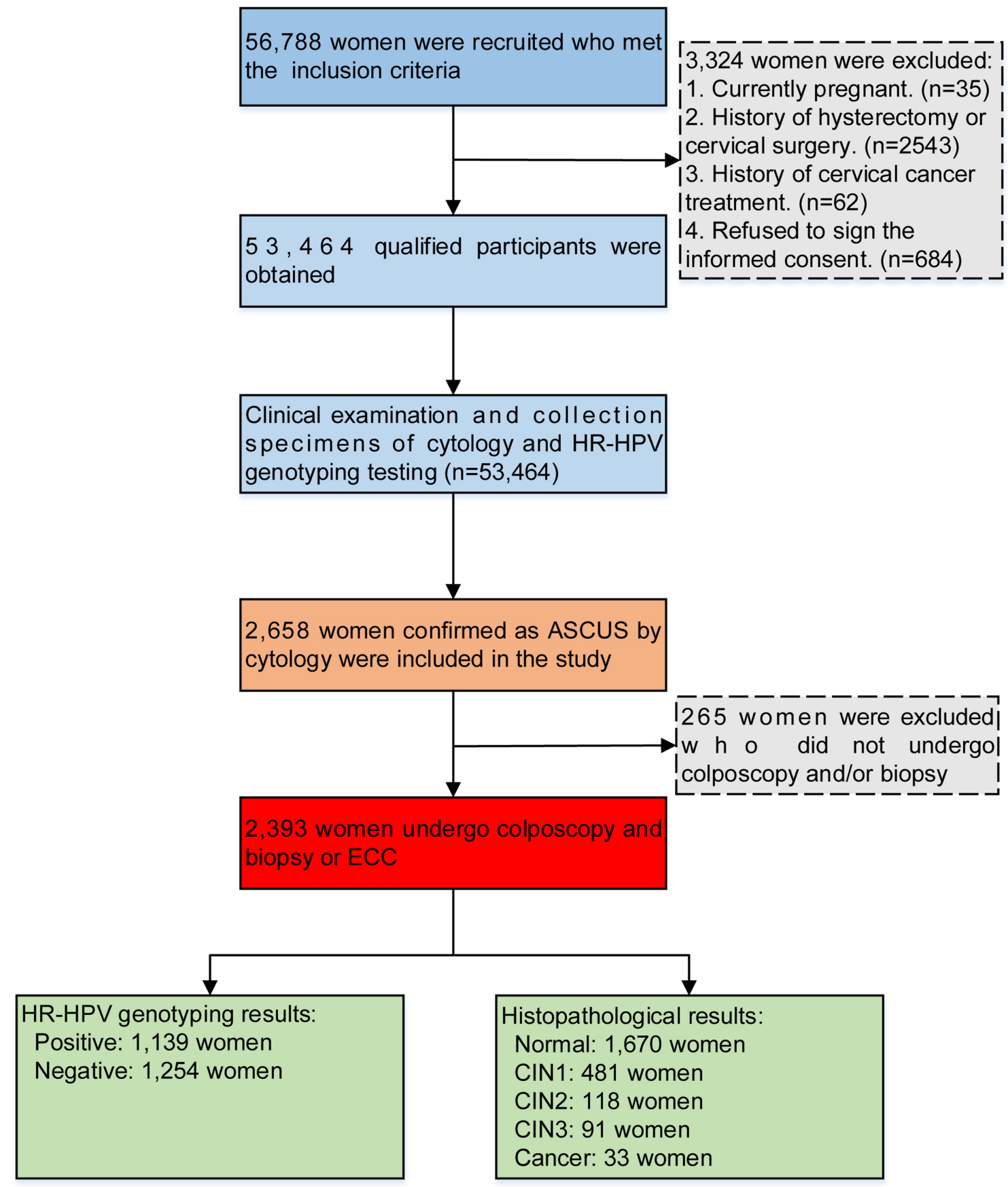

Figure I The flowchart of this study.

Abbreviations: HR-HPV, HR-HPV, high-risk human papillomavirus, including types HPV-16, $-18,-31,-33,-35,-39,-45,-5 \mathrm{I},-52,-56,-58,-59,-66,-68 ;$ ASC-US, atypical squamous cells of undetermined significance; CINI/2/3, cervical intraepithelial neoplasia grade I/2/3; ECC, endocervical curettage.

7.0\%; HPV33, 5.8\%; HPV31, 5.4\%; HPV56, 2.5\%; HPV59, 2.5\%; HPV39, 2.1\%; HPV51, 2.1\%; HPV45, 1.7\%; HPV35, $1.2 \%$; HPV66, $0.8 \%$ and HPV68, $0.8 \%$. The prevalence rate of HR-HPV increased as more HPV genotypes were combined. The combination of HPV16/18/31/33/52/58 was able to identify $91.3 \%(\mathrm{p}<0.001)$ of women whose cytology was ASC-US and who had histologically proven CIN2+, similar to the HR-HPV model (95.0\%). However, based on the HPV16/18 model, the rate was only $54.1 \%$.

\section{Risk of CIN2+ Stratified by HR-HPV Genotype in Women with ASC-US}

We analyzed the factors that may predict $\mathrm{CIN} 2+$ in women with ASC-US, as shown in Table 4. HR-HPV-positive cases 
Table I Characteristics for Women with Cytology Showing ASC-US ( $\mathrm{N}=2393)$

\begin{tabular}{|c|c|c|}
\hline Characteristics & $\begin{array}{l}\text { No. of } \\
\text { Women }\end{array}$ & $\begin{array}{l}\text { Mean (x士s) or } \\
\text { Prevalence (\%) }\end{array}$ \\
\hline Age & 2393 & $38.42 \pm 10.28$ \\
\hline $21-30$ & 605 & $26.33 \pm 2.88$ \\
\hline $31-40$ & 835 & $35.57 \pm 2.88$ \\
\hline $4 I-50$ & 671 & $44.80 \pm 2.71$ \\
\hline $5 I-65$ & 255 & $56.18 \pm 4.25$ \\
\hline$>65$ & 27 & $70.56 \pm 3.52$ \\
\hline \multicolumn{3}{|l|}{ Degree of education } \\
\hline Uneducated & 217 & 9.1 \\
\hline Primary school education & 373 & 15.6 \\
\hline Middle school education & 1188 & 49.6 \\
\hline$\geq$ Higher education & 615 & 25.7 \\
\hline \multicolumn{3}{|l|}{ Drinking history } \\
\hline Yes-At least 2 times a week & 96 & 4.0 \\
\hline Yes- Less than 2 times a week & 660 & 27.6 \\
\hline No & 1637 & 68.4 \\
\hline \multicolumn{3}{|l|}{ Smoking history } \\
\hline Yes-At least 2 times a week & 49 & 2.0 \\
\hline Yes-Less than 2 times a week & 12 & 0.5 \\
\hline No & 2332 & 97.5 \\
\hline \multicolumn{3}{|l|}{ Times of pregnancy } \\
\hline$\leq 2$ & 1538 & 64.3 \\
\hline$>2$ & 855 & 35.7 \\
\hline \multicolumn{3}{|l|}{ Times of childbirth } \\
\hline$\leq 2$ & 2041 & 85.3 \\
\hline$>2$ & 352 & 14.7 \\
\hline \multicolumn{3}{|l|}{ HR-HPV infection } \\
\hline Positive & 1139 & 47.6 \\
\hline Negative & 1254 & 52.4 \\
\hline
\end{tabular}

Abbreviations: ASC-US, atypical squamous cells of undetermined significance; HR-HPV, high-risk human papillomavirus, including types HPV-16, $-18,-31,-33$, $-35,-39,-45,-5 \mathrm{I},-52,-56,-58,-59,-66,-68$.

were associated with 16.18 (95\% confidence interval (CI), 7.87 to $33.24 ; \mathrm{p}<0.001)$ times higher odds of histologic CIN2+ after adjustments for age, education level, smoking, drinking, number of pregnancies and number of childbirths. The risk was highest for CIN2+ among women with ASC-US with HPV16 infection (OR, 26.38; 95\% CI, 18.28 to 38.09; $\mathrm{p}<0.001$ ). HPV18 (OR, 4.44; 95\% CI, 2.34 to 8.42; $<<0.001$ ), HPV31 (OR, 2.97; 95\% CI, 1.37 to $6.45 ; \mathrm{p}=0.006$ ), HPV33 (OR, 3.38; 95\% CI, 1.63 to 7.00; $\mathrm{p}=0.001$ ), HPV52 (OR, 2.96; 95\% CI, t1.96 to 4.48; $<<0.001$ ) and HPV58 (OR, 7.04; 95\% CI, 4.59 to $10.78 ; \mathrm{p}<0.001$ ) were also associated with a higher risk of histologic CIN2+, whereas HPV35, HPV39, HPV45, HPV51, HPV56, HPV59, HPV66, and HPV68 did not have a significant effect on the risk of CIN2+. We further estimated
Table 2 Prevalence of Different HR-HPV Genotypes in Women with ASC-US ( $\mathrm{N}=2393)$

\begin{tabular}{|l|l|l|}
\hline Variate & Positive n (\%) & Negative n (\%) \\
\hline Age $^{\mathrm{a}}$ & & \\
$2 \mathrm{I}-30$ & $310(5 \mathrm{I} .2 \%)$ & $295(48.8 \%)$ \\
$3 \mathrm{I}-40$ & $392(46.9 \%)$ & $443(53.1 \%)$ \\
$4 \mathrm{I}-50$ & $314(46.8 \%)$ & $357(53.2 \%)$ \\
$5 \mathrm{I}-65$ & $112(43.9 \%)$ & $143(56.1 \%)$ \\
$>65$ & $11(40.7 \%)$ & $16(59.3 \%)$ \\
\hline HR-HPV & $1139(47.6 \%)$ & $1254(52.4 \%)$ \\
HPVI6 & $226(9.4 \%)$ & $2167(90.6 \%)$ \\
HPVI8 & $88(3.7 \%)$ & $2305(96.3 \%)$ \\
HPV3I & $64(2.7 \%)$ & $2329(97.3 \%)$ \\
HPV33 & $71(3.0 \%)$ & $2322(97.0 \%)$ \\
HPV35 & $26(1.1 \%)$ & $2367(98.9 \%)$ \\
HPV39 & $69(2.9 \%)$ & $2324(97.1 \%)$ \\
HPV45 & $33(1.4 \%)$ & $2360(98.6 \%)$ \\
HPV5I & $103(4.3 \%)$ & $2290(95.7 \%)$ \\
HPV52 & $329(13.7 \%)$ & $2064(86.3 \%)$ \\
HPV56 & $81(3.4 \%)$ & $2312(96.6 \%)$ \\
HPV58 & $201(8.4 \%)$ & $2192(91.6 \%)$ \\
HPV59 & $62(2.6 \%)$ & $2331(97.4 \%)$ \\
HPV66 & $60(2.5 \%)$ & $2333(97.5 \%)$ \\
HPV68 & $67(2.8 \%)$ & $2326(97.2 \%)$ \\
\hline HPs)
\end{tabular}

Notes: ${ }^{a}$ Only consider the prevalence of HR-HPV.

Abbreviations: HR-HPV, high-risk human papillomavirus, including types HPV-16, $-18,-31,-33,-35,-39,-45,-51,-52,-56,-58,-59,-66,-68$. ASC-US, atypical squamous cells of undetermined significance.

the risk of CIN2+ according to different HPV genotype models among women with ASC-US. The estimated ORs of CIN2 + were 19.34 (95\% CI, 13.76 to $27.19 ; \mathrm{p}<0.001)$ for the HPV16/18 model, 18.73 (95\% CI, 13.46 to 26.07; $<<0.001$ ) for the HPV16/18/58 model, 16.99 (95\% CI, 11.73 to 24.61 ; $\mathrm{p}<0.001)$ for the HPV16/18/52/58 model, 19.76 (95\% CI, 13.22 to 29.55 ; $\mathrm{p}<0.001$ ) for the HPV16/18/33/52/58 model, and 15.08 (95\% CI, 7.61 to $29.86 ; \mathrm{p}<0.001)$ for the HPV16/ $18 / 31 / 33 / 52 / 58$ model.

\section{Effectiveness of Different HR-HPV Genotype Models for the Triage of Women with ASC-US}

The study endpoint was histological detection of CIN2+ at any of the follow-up visits. The sensitivities and NPVs of the different HR-HPV genotype models increased with the increase in the number of combined HPV genotypes, and the highest was the HR-HPV model followed by HPV16/ 18/31/33/52/58, HPV16/18/33/52/58, HPV16/18/52/58, HPV16/18/58 and HPV16/18. However, as the number of HPV genotype combinations increased, the specificity and 
Table 3 Distribution of Different HR-HPV Genotypes Model According to Histologic Diagnosis in Women with ASC-US. $(\mathrm{N}=2393)$

\begin{tabular}{|c|c|c|c|}
\hline \multirow{2}{*}{$\begin{array}{l}\text { HR-HPV } \\
\text { Types Model }\end{array}$} & \multicolumn{2}{|c|}{ Histologic Diagnosis } & \multirow[b]{2}{*}{$P$-value } \\
\hline & $\begin{array}{l}\text { Normal/CINI } \\
\text { N=215I, n (\%) }\end{array}$ & $\begin{array}{l}\text { CIN2+ } \\
N=242, \text { n (\%) }\end{array}$ & \\
\hline HR-HPV & 909 (42.3\%) & $230(95.0 \%)$ & $<0.001$ \\
\hline HPVI6 & $110(5.1 \%)$ & $116(47.9 \%)$ & $<0.001$ \\
\hline HPVI8 & 7I (3.3\%) & 17 (7.0\%) & 0.004 \\
\hline HPV3I & $5 \mathrm{I}(2.4 \%)$ & $13(5.4 \%)$ & 0.006 \\
\hline HPV33 & $57(2.6 \%)$ & 14 (5.8\%) & 0.006 \\
\hline HPV35 & $23(1.1 \%)$ & $3(1.2 \%)$ & 1.000 \\
\hline HPV39 & $64(3.0 \%)$ & $5(2.1 \%)$ & 0.423 \\
\hline HPV45 & $29(1.3 \%)$ & $4(1.7 \%)$ & 0.925 \\
\hline HPV5I & $98(4.6 \%)$ & $5(2.1 \%)$ & 0.070 \\
\hline HPV52 & 284 (13.2\%) & $45(18.6 \%)$ & 0.021 \\
\hline HPV56 & $75(3.5 \%)$ & $6(2.5 \%)$ & $0.4 I I$ \\
\hline HPV58 & $150(7.0 \%)$ & $5 \mathrm{I}(2 \mathrm{I} .1 \%)$ & $<0.001$ \\
\hline HPV59 & $56(2.6 \%)$ & $6(2.5 \%)$ & 0.908 \\
\hline HPV66 & $58(2.7 \%)$ & $2(0.8 \%)$ & 0.078 \\
\hline HPV68 & $65(3.0 \%)$ & $2(0.8 \%)$ & 0.050 \\
\hline$H P V I 6 / 18^{a}$ & $178(8.3 \%)$ & $13 \mid(54.1 \%)$ & $<0.001$ \\
\hline HPVI6/I8/58 & $314(14.6 \%)$ & $174(7 \mid .9 \%)$ & $<0.001$ \\
\hline $\begin{array}{l}\text { HPVI6/I8/52/ } \\
58^{\mathrm{c}}\end{array}$ & $568(26.4 \%)$ & $203(83.9 \%)$ & $<0.001$ \\
\hline $\begin{array}{l}\text { HPVI6/I8/33/ } \\
52 / 58^{d}\end{array}$ & $611(28.4 \%)$ & $212(87.6 \%)$ & $<0.001$ \\
\hline $\begin{array}{l}\text { HPVI6/I8/3I/ } \\
33 / 52 / 58^{\mathrm{e}}\end{array}$ & $645(30.0 \%)$ & $221(91.3 \%)$ & $<0.001$ \\
\hline
\end{tabular}

Notes: ${ }^{a}$ Women with HPVI6 and/or HPVI 8 infection; ${ }^{b}$ all women with any positive of HPVI6, HPVI8, and HPV58; 'all women with any positive of HPVI6, HPVI8, HPV52 and HPV58; 'all women with any positive of HPVI6, HPVI8, HPV33, HPV52 and HPV58; ${ }^{e}$ all women with any positive of HPVI6, HPVI8, HPV3I, HPV33, HPV52 and HPV58.

Abbreviations: HR-HPV, high-risk human papillomavirus, including types HPV-16, $-18,-31,-33,-35,-39,-45,-5 \mathrm{I},-52,-56,-58,-59,-66,-68$; CIN, cervical intraepithelial neoplasia; $\mathrm{CIN2+}$, cervical intraepithelial neoplasia grade 2 or worse; ASC-US, atypical squamous cells of undetermined significance.

PPV decreased. The sensitivity $(91.3 \%$ vs $95.0 \%)$ and NPV $(98.6 \%$ vs $99.0 \%)$ of the HPV16/18/31/33/52/58 model were similar to those of the HR-HPV model for identifying CIN2+ among women with ASC-US, but the specificity $(70.0 \%$ vs $57.7 \%)$ and PPV (25.5\% vs $20.2 \%)$ were higher than those of the HR-HPV model. In addition, the referral rate for the HPV16/18/31/33/52/58 model (36.2\%) was significantly lower than that for the HRHPV model (47.6\%) (Table 5).

\section{Discussion}

ASC-US is the most frequent abnormal cytology result in cervical screening, but most patients do not present cervical
Table 4 Risk of CIN2+ Stratified by HR-HPV Genotype in Women with ASC-US $(\mathrm{N}=2393)$

\begin{tabular}{|c|c|c|c|c|}
\hline $\begin{array}{l}\text { HR-HPV } \\
\text { Types }\end{array}$ & $\begin{array}{l}\text { No. of } \\
\text { Women }\end{array}$ & OR & $\begin{array}{l}\text { OR }_{\text {adjust }} \\
(95 \% \mathrm{Cl})^{\mathrm{a}}\end{array}$ & $P$-value \\
\hline \multicolumn{5}{|l|}{ Age } \\
\hline $21-30$ & 605 & I (R) & I (R) & \\
\hline $31-40$ & 835 & $1.62(1.10-2.38)$ & $2.01(1.28-3.17)$ & 0.003 \\
\hline $4 I-50$ & 671 & $2.16(1.47-3.18)$ & $2.68(1.70-4.22)$ & $<0.001$ \\
\hline $51-65$ & 255 & $1.17(0.67-2.04)$ & $1.56(0.82-2.96)$ & 0.176 \\
\hline$>65$ & 27 & $1.10(0.25-4.80)$ & $1.29(0.17-9.77)$ & 0.805 \\
\hline \multicolumn{5}{|l|}{ HR-HPV } \\
\hline Negative & 1254 & I (R) & I (R) & \\
\hline Positive & 1139 & 26.19 & 16.18 & $<0.001$ \\
\hline & & (14.57-47.08) & $(7.87-33.24)$ & \\
\hline \multicolumn{5}{|l|}{ HPVI6 } \\
\hline Negative & 2167 & I (R) & I (R) & \\
\hline Positive & 226 & 17.08 & 26.38 & $<0.001$ \\
\hline & & $(12.44-23.45)$ & $(18.28-38.09)$ & \\
\hline \multicolumn{5}{|l|}{ HPVI8 } \\
\hline Negative & 2305 & I (R) & I (R) & \\
\hline Positive & 88 & $2.21(1.28-3.82)$ & $4.44(2.34-8.42)$ & $<0.001$ \\
\hline \multicolumn{5}{|l|}{ HPV3I } \\
\hline Negative & 2329 & I (R) & I (R) & \\
\hline Positive & 64 & $2.34(1.25-4.36)$ & $2.97(1.37-6.45)$ & 0.006 \\
\hline \multicolumn{5}{|l|}{ HPV33 } \\
\hline Negative & 2322 & $\mathrm{I}(\mathrm{R})$ & I (R) & \\
\hline Positive & 71 & $2.26(I .24-4.11)$ & $3.38(1.63-7.00)$ & 0.001 \\
\hline \multicolumn{5}{|l|}{ HPV35 } \\
\hline Negative & 2367 & I (R) & I (R) & \\
\hline Positive & 26 & $1.16(0.35-3.90)$ & $2.09(0.58-7.55)$ & 0.262 \\
\hline \multicolumn{5}{|l|}{ HPV39 } \\
\hline Negative & 2324 & I (R) & I (R) & \\
\hline Positive & 69 & $0.69(0.27-1.73)$ & $1.23(0.43-3.52)$ & 0.699 \\
\hline \multicolumn{5}{|l|}{ HPV45 } \\
\hline Negative & 2360 & I (R) & I (R) & \\
\hline Positive & 33 & $1.23(0.43-3.53)$ & $1.32(0.4|-4.3|)$ & 0.642 \\
\hline \multicolumn{5}{|l|}{ HPV5I } \\
\hline Negative & 2290 & I (R) & I (R) & \\
\hline Positive & 103 & $0.44(0.18-1.10)$ & $0.53(0.19-1.50)$ & 0.233 \\
\hline \multicolumn{5}{|l|}{ HPV52 } \\
\hline Negative & 2064 & I (R) & I (R) & \\
\hline Positive & 329 & $1.50(1.06-2.13)$ & $2.96(1.96-4.48)$ & $<0.001$ \\
\hline \multicolumn{5}{|l|}{ HPV56 } \\
\hline Negative & 2312 & I (R) & I (R) & \\
\hline Positive & 81 & $0.70(0.30-1.63)$ & $0.66(0.23-1.89)$ & 0.438 \\
\hline \multicolumn{5}{|l|}{ HPV58 } \\
\hline Negative & 2192 & I (R) & I (R) & \\
\hline Positive & 201 & $3.56(2.51-5.06)$ & $\begin{array}{l}7.04 \\
(4.59-10.78)\end{array}$ & $<0.001$ \\
\hline
\end{tabular}

(Continued) 
Table 4 (Continued).

\begin{tabular}{|c|c|c|c|c|}
\hline $\begin{array}{l}\text { HR-HPV } \\
\text { Types }\end{array}$ & $\begin{array}{l}\text { No. of } \\
\text { Women }\end{array}$ & OR & $\begin{array}{l}\text { OR } R_{\text {adjust }} \\
(95 \% \text { Cl })^{a}\end{array}$ & $P$-value \\
\hline $\begin{array}{l}\text { HPV59 } \\
\text { Negative } \\
\text { Positive }\end{array}$ & $\begin{array}{l}2331 \\
62\end{array}$ & $\begin{array}{l}\text { I (R) } \\
0.95(0.4 \mid-2.23)\end{array}$ & $\begin{array}{l}\text { I (R) } \\
0.76(0.29-2.02)\end{array}$ & 0.582 \\
\hline $\begin{array}{l}\text { HPV66 } \\
\text { Negative } \\
\text { Positive }\end{array}$ & $\begin{array}{l}2333 \\
60\end{array}$ & $\begin{array}{l}\text { I (R) } \\
0.30(0.07-1.24)\end{array}$ & $\begin{array}{l}\text { I (R) } \\
0.14(0.02-0.85)\end{array}$ & 0.032 \\
\hline $\begin{array}{l}\text { HPV68 } \\
\text { Negative } \\
\text { Positive }\end{array}$ & $\begin{array}{l}2326 \\
67\end{array}$ & $\begin{array}{l}\text { I (R) } \\
0.27(0.07-1.10)\end{array}$ & $\begin{array}{l}\text { I (R) } \\
0.22(0.05-1.02)\end{array}$ & 0.053 \\
\hline $\begin{array}{l}\text { HPVI6/18b } \\
\text { Negative } \\
\text { Positive }\end{array}$ & $\begin{array}{l}2084 \\
309\end{array}$ & $\begin{array}{l}\text { I (R) } \\
\text { I3.08 } \\
(9.73-17.58)\end{array}$ & $\begin{array}{l}\text { I (R) } \\
19.34 \\
(13.76-27.19)\end{array}$ & $<0.001$ \\
\hline $\begin{array}{l}\text { HPVI6/18/ } \\
58^{\mathrm{c}} \\
\quad \text { Negative } \\
\text { Positive }\end{array}$ & $\begin{array}{l}1905 \\
488\end{array}$ & $\begin{array}{l}\text { I (R) } \\
\text { I4.97 } \\
(11.04-20.30)\end{array}$ & $\begin{array}{l}\text { I (R) } \\
18.73 \\
(13.46-26.07)\end{array}$ & $<0.001$ \\
\hline $\begin{array}{l}\text { HPVI6/18/ } \\
52 / 58^{\mathrm{d}} \\
\text { Negative } \\
\text { Positive }\end{array}$ & $\begin{array}{l}1622 \\
771\end{array}$ & $\begin{array}{l}I(R) \\
14.5 I \\
(10.16-20.7 I)\end{array}$ & $\begin{array}{l}\text { I (R) } \\
16.99 \\
(11.73-24.61)\end{array}$ & $<0.001$ \\
\hline $\begin{array}{l}\text { HPVI6/18/ } \\
33 / 52 / 58^{\mathrm{e}} \\
\text { Negative } \\
\text { Positive }\end{array}$ & $\begin{array}{l}1570 \\
823\end{array}$ & $\begin{array}{l}I(R) \\
17.81 \\
(I 2.02-26.40)\end{array}$ & $\begin{array}{l}\text { I (R) } \\
19.76 \\
(13.22-29.55)\end{array}$ & $<0.001$ \\
\hline $\begin{array}{l}\text { HPVI6/18/ } \\
31 / 33 / 52 / 58^{f} \\
\text { Negative } \\
\text { Positive }\end{array}$ & $\begin{array}{l}1527 \\
866\end{array}$ & $\begin{array}{l}\text { I (R) } \\
24.57 \\
(I 5.56-38.8 I)\end{array}$ & $\begin{array}{l}\text { I (R) } \\
\text { I5.08 } \\
(7.6 I-29.86)\end{array}$ & $<0.001$ \\
\hline
\end{tabular}

Notes: ${ }^{a} O R$ values were adjusted for age, education level, smoking, drinking, number of pregnancy, and number of childbirth; ${ }^{b}$ women with HPVI6 and/or HPVI8 infection directly referred to colposcopy and biopsy; ${ }^{c}$ all women with any positive of HPVI6, HPVI8, and HPV58 were directly referred to colposcopy and biopsy; ${ }^{d}$ all women with any positive of HPVI6, HPVI8, HPV52 and HPV58 were directly referred to colposcopy and biopsy; ${ }^{\mathrm{e} a l l}$ women with any positive of HPVI6, HPV18, HPV33, HPV52 and HPV58 were directly referred to colposcopy and biopsy; fall women with any positive of HPVI6, HPVI8, HPV3I, HPV33, HPV52 and HPV58 were directly referred to colposcopy and biopsy.

Abbreviations: OR, odds ratio; ASC-US, atypical squamous cells of undetermined significance; HR-HPV, high-risk human papillomavirus, including types HPV-16, -18 , $-3 \mathrm{I},-33,-35,-39,-45,-5 \mathrm{I},-52,-56,-58,-59,-66,-68$; $\mathrm{R}$, reference.

lesions or are only diagnosed with CIN1 during follow-up, and less than $10 \%$ of women progress to $\mathrm{CIN} 2 / 3$ or cancer. ${ }^{8}$ Thus, deciding the appropriate clinical management strategy for women with ASC-US is a problem that needs a solution. HR-HPV testing has been included in the programs of cervical cancer screening in recent years, which can both be used to triage patients with ASC-US ${ }^{2,17}$ and be a part of screening combined with the TCT. The current management strategy is that all ASC-US women with an HR-HPVpositive result are immediately referred for colposcopy/ biopsy. ${ }^{18}$ Different HR-HPV genotypes are associated with different levels of risk for the progression of CIN to cervical cancer. The effectiveness of specific HR-HPV types in the triage of women with ASC-US may vary with the different combination of different HPV genotypes. Thus, it is meaningful to identify the specific HR-HPV genotype model with higher sensitivity and specificity and lower referral rates to triage women with ASC-US.

Previous reports ${ }^{13,19}$ mainly evaluated the performance of HR-HPV or HPV16/18 genotyping in triaging patients with ASC-US. Nevertheless, data on HR-HPV types other than HPV16 and HPV18 are rare and inconsistent. This study evaluated the triage effectiveness of all HR-HPV genotypes in women with ASC-US, and we discuss the triage efficiency of different HR-HPV genotype models (HR-HPV, HPV16/18, HPV16/18/58, HPV16/18/52/58, HPV16/18/31/52/58, HPV16/18/31/33/52/58) for patients with ASC-US from a Chinese population whose biopsies proved to be CIN2+. We found that the HPV16/18/31/33/ $58 / 52$ model has a relatively high sensitivity, specificity, PPV, and NPV but a lower referral rate than the HR-HPV model or other HR-HPV genotype models.

The rate of ASC-US findings by cervical cytology ranged from $3 \%$ to $10 \%$ in previous studies. ${ }^{8,20-22}$ In line with these results, our research found that ASC-US accounted for 5.0\% of all cytology results. However, the prevalence of HR-HPV among women with ASC-US was quite inconsistent in different studies. Although one study reported that the prevalence rate of HR-HPV among women with ASC-US was $41.7 \%,{ }^{23}$ another study reported a prevalence rate of $23 \%{ }^{24}$ The prevalence rate of HR-HPV among patients with ASCUS was $47.6 \%$ in our research. Moreover, among patients with ASC-US whose biopsies proved to be CIN2+, the infection rates of HPV16, HPV18, HPV31, HPV33, HPV52 and HPV58 were $47.9 \%, 7.0 \%, 5.4 \%, 5.8 \%, 18.6 \%$ and $21.1 \%$, respectively. These findings suggest a higher possibility of progression to high-grade CIN in patients with ASC-US who are simultaneously infected with HPV16, HPV18, HPV31, HPV33, HPV52 or HPV58, which implies the significance of specific HPV genotype detection.

Previous studies compared the accuracy of the HR-HPV test with repeated cytology for potential CIN2+ or CIN3+ in patients with ASC-US. The results indicated that the HR-HPV 
Table 5 The Effect of Different HR-HPV Genotypes Model in Triaging Women with ASC-US. (N=2393)

\begin{tabular}{|c|c|c|c|c|c|}
\hline $\begin{array}{l}\text { HR-HPV Genotypes } \\
\text { Model }\end{array}$ & $\begin{array}{l}\text { Sensitivity \% (95\% } \\
\text { CI) }\end{array}$ & $\begin{array}{l}\text { Specificity \% (95\% } \\
\text { Cl) }\end{array}$ & $\begin{array}{l}\text { PPV \% (95\% } \\
\text { CI) }\end{array}$ & $\begin{array}{l}\text { NPV \% }(95 \% \\
\text { CI) }\end{array}$ & $\begin{array}{l}\text { Referral Rate } \%(n / \\
\text { N) }\end{array}$ \\
\hline HR-HPV & $95.0(92.3-97.8)$ & $57.7(55.7-59.8)$ & $20.2(18.1-22.7)$ & $99.0(98.5-99.6)$ & $47.6(1139 / 2393)$ \\
\hline HPVI6/18 & $54.1(47.9-60.4)$ & $91.7(90.6-92.9)$ & $42.4(36.5-47.5)$ & $94.7(93.3-95.3)$ & $12.9(309 / 2393)$ \\
\hline HPVI $6 / 18 / 58^{d}$ & $71.9(66.2-77.6)$ & $85.4(83.9-86.9)$ & $35.7(31.1-39.6)$ & $96.4(95.1-96.9)$ & $20.4(488 / 2393)$ \\
\hline HPVI6/I8/52/58 & $83.9(79.3-88.5)$ & $73.6(71.7-75.5)$ & $26.3(22.9-29.1)$ & $97.6(96.2-97.8)$ & $32.2(77 I / 2393)$ \\
\hline HPVI6/I8/33/52/58 & $87.6(83.5-91.8)$ & $71.6(69.7-73.5)$ & $25.8(22.5-28.5)$ & 98.1 (96.7-98.2) & $34.4(823 / 2393)$ \\
\hline HPVI6/I8/3I/33/52/58 & $91.3(87.8-94.9)$ & $70.0(68.1-72.0)$ & $25.5(22.4-28.2)$ & $98.6(97.3-98.7)$ & $36.2(866 / 2393)$ \\
\hline
\end{tabular}

Notes: ${ }^{a}$ The rate of referred to colposcopy in ASC-US women; ball women with any positive of HPVI6, HPVI8, HPV 3I, HPV 33, HPV 35, HPV 39, HPV 45, HPV 5I, HPV 52, HPV 56, HPV 58, HPV 59, HPV 66 and HPV68 were directly referred to colposcopy and biopsy; 'women with HPVI6 and/or HPVI8 infection directly referred to colposcopy and biopsy; ${ }^{\mathrm{d}}$ all women with any positive of HPVI6, HPVI8, and HPV58 were directly referred to colposcopy and biopsy; ${ }^{\mathrm{e}}$ all women with any positive of HPVI6, HPVI8, HPV52 and HPV58 were directly referred to colposcopy and biopsy; fall women with any positive of HPVI6, HPVI8, HPV33, HPV52 and HPV58 were directly referred to colposcopy and biopsy; ${ }^{g}$ all women with any positive of HPVI6, HPVI8, HPV3I, HPV33, HPV52 and HPV58 were directly referred to colposcopy and biopsy. Abbreviations: HR-HPV, high-risk human papillomavirus, including types HPV-I6, -18, $-31,-33,-35,-39,-45,-5 I,-52,-56,-58,-59,-66,-68$; PPV, positive predictive value; NPV, negative predictive value.

test is more sensitive than repeated cytology in the triage of patients with ASC-US, but there is no significant difference in the specificity. ${ }^{2}$ Our study shows that the sensitivity of HRHPV to detect CIN2+ among patients with ASC-US is $95.0 \%$ but that the specificity is only $57.7 \%$. Therefore, triaging patients with ASC-US with the recommended HR-HPV test will increase the referral rates of colposcope, unnecessary costs and burden to patients. In our previous study, ${ }^{25}$ HPV16, HPV18, HPV31, HPV33, HPV52 and HPV58 were the most common genotypes in women with HSILs in China. Other studies ${ }^{26}$ have also indicated that the prevalence rates of HPV16, HPV18, HPV31, HPV33, HPV52 and HPV58 among patients with ASC-US with detected CIN2 or CIN3 are higher. This is consistent with our research, and we found that HPV16, HPV18, HPV31, HPV33, HPV52 and HPV58 were the most prevalent among patients with ASC-US with a histologic diagnosis of CIN2+. Therefore, the risk of different HPV genotypes associated with CIN2+ among patients with ASC-US was estimated. We found that HR-HPV positivity was associated with an increased odds of CIN2+ histology (OR, 16.18; 95\% CI, 7.87 to 33.24). In the analysis of different HR-HPV genotypes, the risk of CIN2+ was highest among women with HPV16 infections. HPV18, HPV31, HPV33, HPV52 and HPV58 were also associated with the risk of histologically proven CIN2+, whereas HPV35, HPV39, HPV45, HPV51, HPV56, HPV59, HPV66, and HPV68 did not have a significant effect on the risk. An increased risk for CIN2+ was observed when the model combined more genotypes. The estimated OR of HPV16/18/58/52/ 31/33 was 15.08 (7.61 to 29.86) according to our research.

Therefore, immediate colposcopy/biopsy of women with ASC-US who are positive for HPV16/18/58/52/31/ 33 may be an alternative strategy. We calculated the sensitivity, specificity, PPV, and NPV of each HPV genotype combination model, including HPV16/18, HPV16/18/ 58, HPV16/18/52/58, HPV16/18/31/52/58, HPV16/18/31/ $33 / 52 / 58$ and HR-HPV, to identify potential CIN2+ populations among patients with ASC-US. Similar to previous results, ${ }^{27}$ HPV16/18 was much more specific than the HPV16/18/31/33/52/58 combination (91.7\% vs $70.0 \%)$ but lost sensitivity $(54.1 \%$ vs $91.3 \%)$, which implies a higher possibility of misdiagnosis. However, we found that the sensitivity of HPV16/18/31/33/52/58 for identifying CIN2+ in women with ASC-US was similar to that of the HR-HPV model but higher than that of HPV16/18/33/ $52 / 58$, HPV16/18/52/58, or HPV16/18/58. The specificity of the HPV16/18/31/33/52/58 model was higher than that of the HR-HPV model (70.0\% vs $57.7 \%)$. Moreover, according to the current guidelines for cervical cancer screening, HR-HPV-positive patients with ASC-US are immediately referred for colposcopy, and the referral rate of HPV16/18/31/33/52/58 was significantly lower than that recommended for HR-HPV (36.2\% vs $47.6 \%)$, which can effectively reduce the high burden of colposcopy referrals. Therefore, it is reasonable to believe that the HPV16/18/31/33/52/58 genotype model is an alternative triage strategy to identify CIN2+ among patients with ASC-US in China.

Potential limitations are that only 1 case of cervical adenocarcinoma was identified in this study, and the study cannot conclude the role of HPV genotyping in the detection of cervical adenocarcinoma. This will impact conclusions regarding the importance of specific genotypes such as HPV45, which are relatively common in cervical adenocarcinomas but less common in CIN2/3 lesions. ${ }^{15}$ In addition, this research was conducted in a single region, 
and we did not perform verification in other regions; thus, it may not be generalizable to other places. Last, this study was not based on a large-scale follow-up, which means it may not reflect the actual screening population.

\section{Conclusion}

This study evaluated the effectiveness of type-specific HRHPV genotypes in the triage of patients with ASC-US in the largest population to date. Due to its high sensitivity and specificity and minimal number of HR-HPV genotypes, we suggest the HPV16/18/31/33/58/52 genotype model as an alternative strategy for the triage of patients with ASC-US. Developing non-16/18 HPV genotyping assays could significantly increase the cost effectiveness of triaging women with ASC-US. To validate our results, further work is needed to evaluate our novel HPV genotype model in external populations.

\section{Abbreviations}

HR-HPV, high-risk human papillomavirus; ASC-US, atypical squamous cells of undetermined significance; LSIL, low-grade squamous intraepithelial lesion; CIN, cervical epithelial neoplasia; TCT, liquid-based cytology; DNA, deoxyribonucleic acid; ECC, endocervical curettage; PPV, positive predictive value; NPV, negative predictive value; OR, odds ratio.

\section{Ethics Approval}

This study was conducted in accordance with the Declaration of Helsinki, and was approved by the Ethics Committees of the Fujian Maternity and Child Health Hospital (2014-045).

\section{Acknowledgments}

The authors would like to thank the Fujian Province Cervical Lesions Screening Cohorts Investigators for planning and conducting the trial and for providing the biological specimens and data for the present study. Above all, we are grateful to the patients who made this study possible.

\section{Author Contributions}

All authors made a significant contribution to the work reported, whether that is in the conception, study design, execution, acquisition of data, analysis and interpretation, or in all these areas; took part in drafting, revising or critically reviewing the article; gave final approval of the version to be published; have agreed on the journal to which the article has been submitted; and agree to be accountable for all aspects of the work.

\section{Funding}

This work was supported by grants from the Fujian Provincial Natural Science Foundation of China (grant no. 2017J01232) and the Fujian Maternity and Child Health Hospital Natural Science Foundation (grant no. YCXM18-18).

\section{Disclosure}

The authors declare that they have no competing interests for this work.

\section{References}

1. Torre LA, Bray F, Siegel RL, et al. Global cancer statistics, 2012. CA Cancer J Clin. 2015;65:87-108.

2. Arbyn M, Roelens J, Simoens C, et al. Human papillomavirus testing versus repeat cytology for triage of minor cytological cervical lesions. Cochrane Database Syst Rev. 2013;3:CD008054.

3. Wright T, Cox J, Massad L, et al. ASCCP Sponsored Consensus Conference. Consensus guidelines for the management of women with cervical cytological abnormalities. JAMA. 2002;287:2120-2129.

4. Koh WJ, Abu-Rustum NR, Bean S, et al. Cervical cancer, version 3. 2019, NCCN clinical practice guidelines in oncology. J Natl Compr Canc Netw. 2019;17:64-84.

5. Cox JT. Management of women with cervical cytology interpreted as ASC-US or as ASC-H. Clin Obstet Gynecol. 2005;48:160-177.

6. Solomon D, Davey D, Kurman R, et al. The 2001 Bethesda System: terminology for reporting results of cervical cytology. JAMA. 2002;287:2114-2119.

7. Perkins Rebecca B, Guido Richard S, Castle Philip E, et al. 2019 ASCCP risk-based management consensus guidelines for abnormal cervical cancer screening tests and cancer precursors. J Low Genit Tract Dis. 2020;24:102-131.

8. Cuzick J, Myers O, Lee JH, et al. Outcomes in women with cytology showing atypical squamous cells of undetermined significance with vs without human papillomavirus testing. JAMA Oncol. 2017;3:1327-1334.

9. Kyrgiou M, Kalliala IE, Mitra A, et al. Immediate referral to colposcopy versus cytological surveillance for minor cervical cytological abnormalities in the absence of HPV test. Cochrane Database Syst Rev. 2017;1:CD009836.

10. Katki HA, Schiffman M, Castle PE, et al. Benchmarking CIN 3+ risk as the basis for incorporating HPV and Pap cotesting into cervical screening and management guidelines. $J$ Low Genit Tract Dis. 2013;17:S28-S35.

11. Schlecht NF, Kulaga S, Robitaille J, et al. Persistent human papillomavirus infection as a predictor of cervical intraepithelial neoplasia. JAMA. 2001;286:3106-3114.

12. Nobbenhuis MA, Walboomers JM, Helmerhorst TJ, et al. Relation of human papillomavirus status to cervical lesions and consequences for cervical-cancer screening: a prospective study. Lancet. 1999;354:20-25.

13. Meijer CJLM, Snijders PJF. Human papillomavirus triage of women with atypical squamous cells of undetermined significance-reduction of overtreatment needed. JAMA. 2017;3:1310-1311.

14. Bouvard V, Baan R, Straif $\mathrm{K}$, et al. A review of human carcinogens-Part B: biological agents. Lancet Oncol. 2009;10:321-322. 
15. de Sanjose S, Quint WG, Alemany L, et al. Human papillomavirus genotype attribution in invasive cervical cancer: a retrospective cross-sectional worldwide study. Lancet Oncol. 2010;11:1048-1056.

16. Kjær SK, Frederiksen K, Munk C, et al. Long-term absolute risk of cervical intraepithelial neoplasia grade 3 or worse following human papillomavirus infection: role of persistence. J Natl Cancer Inst. 2010;102:1478-1488.

17. Lee S, Kim JW, Hong JH, et al. Clinical significance of HPV DNA cotesting in Korean women with ASCUS or ASC-H. Diagn Cytopathol. 2014;42:1058-1062.

18. Saslow D, Solomon D, Lawson HW, et al. American Cancer Society, American Society for Colposcopy and Cervical Pathology, and American Society for Clinical Pathology screening guidelines for the prevention and early detection of cervical cancer. $C A$ Cancer $J$ Clin. 2012;62:147-172.

19. Lin CQ, Cui JF, Zhang X, et al. Human papillomavirus genotyping to predict the risk of cervical precancerous lesions or cancer in women with minor abnormal cytology in China. Acta Cytol. 2015;59:405-411.

20. Guo Z, Jia MM, Chen Q, et al. Performance of different combination models of high-risk HPV genotyping in triaging chinese women with atypical squamous cells of undetermined significance. Front Oncol. 2019;9:902.

21. Zheng B, Yang H, Li Z, et al. Atypical squamous cells of undetermined significance cervical cytology report rate and histologic follow-up findings from the largest College of American Pathologists-Certified Laboratory in China. Arch Pathol Lab Med. 2019;143:748-752.
22. Kurman RJ, Henson DE, Herbst AL, et al. Interim guidelines for management of abnormal cervical cytology. The 1992 national cancer institute workshop. JAMA. 1994;271:1866-1869.

23. Risley C, Clarke MA, Geisinger KR, et al. Racial differences in HPV type 16 prevalence in women with ASCUS of the uterine cervix. Cancer Cytopathol. 2020.

24. Hashim D, Engesæter B, Baadstrand Skare G, et al. Real-world data on cervical cancer risk stratification by cytology and HPV genotype to inform the management of HPV-positive women in routine cervical screening. Br J Cancer. 2020.

25. Sun P, Song Y, Ruan G, et al. Clinical validation of the PCR-reverse dot blot human papillomavirus genotyping test in cervical lesions from Chinese women in the Fujian province: a hospital-based population study. J Gynecol Oncol. 2017;28:e50.

26. Kang WD, Ju UC, Kim SM. Is human papillomavirus genotype important in predicting disease progression in women with biopsy-proven negative or CIN1 of atypical squamous cell of undetermined significance (ASC-US) cytology? Gynecol Oncol. 2018;148:305-310.

27. Silver MI, Andrews J, Cooper CK, et al. Risk of cervical intraepithelial neoplasia 2 or worse by cytology, human papillomavirus 16/18, and colposcopy impression: a systematic review and meta-analysis. Obstet Gynecol. 2018;132:725-735.
Risk Management and Healthcare Policy

\section{Publish your work in this journal}

Risk Management and Healthcare Policy is an international, peerreviewed, open access journal focusing on all aspects of public health, policy, and preventative measures to promote good health and improve morbidity and mortality in the population. The journal welcomes submitted papers covering original research, basic science, clinical \& epidemiological studies, reviews and evaluations,
Dovepress

guidelines, expert opinion and commentary, case reports and extended reports. The manuscript management system is completely online and includes a very quick and fair peer-review system, which is all easy to use. Visit http://www.dovepress.com/testimonials.php to read real quotes from published authors. 